\title{
APPROACH IN NONLINEAR DESCRIPTOR SYSTEM USING OUTPUT FEEDBACK
}

\author{
ELMER ROLANDO LLANOS VILLARREAL*, ALEX SANDRO DE ARAUJO SILVA ${ }^{\dagger}$, \\ SAMEQUE FARIAS CUNHA DE OLIVEIRA $\ddagger$ \\ * Universidade Federal Rural do Semi-Árido (UFERSA) \\ Departamento de Ciências Exatas e Naturais (DCEN) \\ Programa de Pós-graduação em Sistemas de Comunicação e Automação \\ Av. Francisco Mota 572 Mossoró, RN, Brasil. \\ †Universidade Federal Rural do Semi-Árido (UFERSA) \\ Departamento de Ciências Exatas, Tecnológicas- Humanas (DCETH) \\ Rua Gamaliel Martins Bezerra,s/n Angicos, RN, Brasil. \\ †Programa de Pós-graduação em Sistemas de Comunicação e Automação/ UFERSA \\ Mossoró, RN, Brasil. \\ Emails: elmerllanos@ufersa.edu.br, alex.araujo@ufersa.edu.br, \\ samequefarias@hotmail.com
}

\begin{abstract}
The present paper study the Lipschitz nonlinear descriptor system. Considering the nonlinear fault-tolerant control system can be made solvable, causal, asymptotically stable. The main result provided condition for the design the output feedback controller, is presented using an LMI approach, where the theorem for existence the output feedback matrix is presented.
\end{abstract}

Keywords — Nonlinear Descriptor System; Output Feedback; LMI.

\section{INTRODUCTION}

The determination of procedures for obtaining the feedback matrix in output feedback control to asymptotically stabilize linear systems is actually an open problem. Several methods have been proposed in the literature based on Lyapunov and Ricatti linear matrix inequalities, or eigenstructure assignment (Fletcher, 1988), (Alexandridis and Paraskevopoulos, 1996),(Castelan et al., 2003).

Research into fault detection and diagnosis for dynamic system has long been recognized as one of the important aspects in practical control systems Several works on the fault detection and diagnosis for nonlinear descriptor systems can be found in (Vemuri et al., 2001), (Chen et al., 2003). In the work in (Gao and Ho, 2006) is presented LMIs technique for a class of Lipschitz nonlinear descriptor systems.

In this paper is presented the approach is developed for nonlinear Lipschitz descriptor systems, thus the show the existence the output feedback matrix, based the paper in (Gao and Ding, 2007). The consider faults here may be unbounded, thus the plant may fail in the presence of faults. This motivates us to investigate faulttolerant control topic, which is very important in many practical systems. However, to the best of our knowledge, very few effort has been made to investigate fault-tolerant control for non linear descriptor systems. In this study, based on the $L M I$ technique and by using the estimated output and faults. The solvability, causality, asymptotic stability and performance are guaranteed. Moreover, the present fault- tolerant controller is outputspace dynamic controller with original coefficient matrices, and it is thus realiable in computations.

\section{SYSTEM DESCRIPTION}

The original mathematical description of a system often consists of a set of differential and algebraic equations. However, in most literature on control theory it is assumed that the algebraic equations can be used to eliminate some variables. The result is a system description consisting only of differential equations that can be written in statespace form as

$$
\dot{x}=F(t, x, u)
$$

\section{PRELIMINARIES AND PROBLEM STATEMENT}

In the present paper, the notations are rather standard. $R$ denotes the set of real numbers; $C$ denotes the complex plane; $R_{e}(a)$ denotes the real part of the complex number $a ; A^{\dagger}$ denotes the generalized inverse of $A ; \lambda_{i}(A)$ denotes the $i$ th eigenvalue de $A ; I_{m}$ denotes an identity matrix with the dimension $m \times m ; O_{n \times p}$ denotes a $n \times p$ matrix with zero entries; $P>0$ (or $P<0$ ) indicates the symmetric matrix $P$ is positive (or negative) definite; $\forall$ means "for all" ; |.|denotes the standard norm symbol ; $L_{2}\left[0 T_{f}\right]$ represents the set of all signals which are square integrable and satisfy $\int_{0}^{T_{f}} d^{\prime}(\tau) d(\tau) d \tau<\infty$; and $\|d\|_{T_{f}}:=$ $\left(\int_{0}^{T_{f}} d^{\prime}(\tau) d(\tau) d \tau\right)^{2}$. The considered nonlinear descriptor systems are described by :

$$
\begin{aligned}
E \dot{x}(t) & =A x(t)+B u(t)+\phi(t, x, u)+B_{d} d+B_{f} f(2) \\
y(t) & =C x(t)
\end{aligned}
$$

where: $x \in \Re^{n}$, is the descriptor state vector; $u \in \Re^{m}, y \in \Re^{p}$ are, respectively, the control input and measurement output vectors and $E \in \Re^{n \times n}$, 
$\operatorname{rank}(E)=q<n$; as the other matrices is an appropriate size with $\operatorname{rank}(B)=m, \operatorname{rank}(C)=p$; $\phi(t, x, u) \in \Re^{n}$ is the real nonlinear vector function satisfying the following

$$
\begin{array}{r}
\|\phi(t, \tilde{x}, u)-\phi(t, x, u)\| \leq\|U(\tilde{x}-x)\| \forall(t, \tilde{x}, u), \\
(t, x, u) \in \Re \times \Re^{n} \times \Re^{m}
\end{array}
$$

and $U \in \Re^{n \times n}$ is known constant matrix. Provided that

$$
\operatorname{rank}\left[U^{\prime} C^{\prime}\right]^{\prime}=\operatorname{rank}(C),
$$

there exists a matrix $K=U C^{\dagger}$ such that

$$
U=K C .
$$

Substitution of (5) into (3) yields

$$
\begin{array}{r}
\|\phi(t, \tilde{x}, u)-\phi(t, x, u)\| \leq\|K C(\tilde{x}-x)\| \leq \\
\theta_{0}\|C(\tilde{x}-x)\| \leq \theta\|\tilde{x}-x\|
\end{array}
$$

$\forall(t, \tilde{x}, u),(t, x, u) \in \Re \times \Re^{n} \times \Re^{m}$

where $\theta_{0}$ and $\theta$ are both positive scalars. In this paper, plant (2) satisfies Lipschitz constraint (6).

\section{BASIC CONCEPTS}

The pair $(E, A)$ is called regular if there exists $s \in \mathcal{C}$ such that $\operatorname{det}(s E-A) \neq 0$ Thus a regular descriptor system is in (Verghese et al., 1981) and (Dai, 1989).

i) stable if all finite roots of $\operatorname{det}(s E-A)=0$ are in the open left half complex plane; havior;

i) impulse free if it exhibits no impulse be-

iii) finite dynamics detectable if there exists $L$ such that $(E, A+L C)$ is regular ans stable;

iv) impulse observable if there exists $L$ such that $(E, A+L C)$ is regular and impulse-free;

v) finite dynamics stabilizable if there exists $F$ such that $(E, A+B F)$ is regular and stable; $(E, A, B)$ is finite dynamics stabilizable if $\operatorname{rank}[s E-A B]=n, \operatorname{Re}[s] \geq 0$.

vi) impulse controllable if there exists $F$ such that $(E, A+B F)$ is regular and impulse-free. If there exists a $F$ such that $(E, A+B F)$ has no impulsive then $(E, A, B)$ is called impulse controllable.

\section{MAIN RESULT FOR NONLINEAR SYSTEM}

Consider the nonlinear system

$$
\begin{aligned}
E \dot{x} & =A x+B u+\phi(t, x, u)+B_{d} d+B_{f} f \\
y(t) & =C x(t)
\end{aligned}
$$

In form basic it is find an static output feedback control law $u(t)=G y(t)$ such that the closed-loop system

$$
E \dot{x}(t)=(A+B G C) x(t)
$$

is $S$-stable: regular, assymptotically stable and free impulses.

\section{Consider}

$$
\begin{aligned}
E \dot{x} & =(A+B G C) u+\phi(t, x, u)+B_{d} d+B \tilde{G} C \tilde{e}(9) \\
y & =C x
\end{aligned}
$$

Now we begin to discuss how to choose $G \in$ $\Re^{p \times m}$ to make the plant (9) satisfy the following:

1) The plant (9) is solvable, causal and asymptotically stable.

2) $\forall T_{f} \geq 0$, the $L_{2}$-gain from the disturbance input $d$ to the system output $y$ is less than or equal to a prescribed $H_{\infty}$ performance $\lambda>0$, i.e.,

$$
\|y\|_{T_{f}} \leq \lambda\|d\|_{T_{f}}
$$

We make a further assumption on $\phi(t, x, u) \in \Re^{n}$, i.e. $\phi(t, 0,0)=0$. Thus, from the Lipschitz constraint (6) one further has

$\|\phi(t, x, u)\| \leq \theta\|x\|, \forall(t, x, u) \in \Re \times \Re^{n} \times \Re^{m}$

Now we have the following statement

Theorem 1 The closed-loop system (9) is solvable, causal and asymptotically stable, and $\|y\|_{T_{f}} \leq \lambda\|d\|_{T_{f}}$ if there exists a matrix $P \in \Re^{n \times n}$ and $G \in \Re^{m \times p}$ such that

$$
\begin{array}{r}
E^{\prime} P=P^{\prime} E \geq 0 \\
(A+B G C) P+P(A+B G C)^{\prime}+I+C^{\prime} C+ \\
\frac{1}{\lambda^{2}} P^{\prime} B_{d} B_{d}^{\prime} P<0
\end{array}
$$

\section{Proof:}

The proof is composed of three parts such as (i) the proof of solvability, (ii) the proof of the asymptotic stability, and (iii) the proof of the guaranteed performance index.

(i) The proof of solvability: It is clear that (13) implies

$\Lambda=(A+B G C)^{\prime} P+P^{\prime}(A+B G C)+I+\theta^{2} P^{\prime} P<0$

and further indicates

$$
(A+B G C)^{\prime} P+P^{\prime}(A+B G C)<0
$$

Moreover, (12) and (15) indicate that the pair ( $E, A+B G C$ ) is casual (or impulse free), asymptotically stable, and $P$ is nonsingular.

Similar can find non-singular matrices $M=$ $\left[\begin{array}{cc}M_{s}^{\prime} & M_{f}^{\prime}\end{array}\right]^{\prime}$ and $N=\left[\begin{array}{ll}N_{s} & N_{f}\end{array}\right]$ such that

$$
\begin{gathered}
M E N=\left[\begin{array}{cc}
I_{q} & 0 \\
0 & 0
\end{array}\right], \mathrm{q}=\operatorname{rank}(\mathrm{E}) \\
M(A+B G C) N=\left[\begin{array}{cc}
A_{s} & 0 \\
0 & -I_{n-q}
\end{array}\right] \\
M^{\prime-1} P N=\left[\begin{array}{cc}
P_{11} & 0 \\
P_{21} & P_{22}
\end{array}\right], P_{11}=P_{11}^{\prime}>0 \\
\left\|M_{f}\right\| \leq 1,\left\|N_{f}\right\|<\frac{1}{\theta \sqrt{1+\epsilon}}
\end{gathered}
$$

where $\epsilon$ is a sufficient small positive number. Let $N^{-1} x=\left[\begin{array}{ll}x_{s}^{\prime} & x_{f}^{\prime}\end{array}\right]^{\prime}$, the descriptor state equation of the plant (9) can be transformed into

$$
\left\{\begin{array}{l}
\dot{x}_{s}=A_{s} x_{s}+M_{s}\left[\phi(t, x, u)+B_{d} d+B \bar{G} C \bar{e}\right] \\
x_{f}=M_{f}\left[\phi(t, x, u)+B_{d} d+B \bar{G} C \bar{e}\right]
\end{array}\right.
$$


With the error dynamic equation can be characterized as

$$
\bar{e}=\bar{x}-x
$$

By using (21) and (20), the closed-loop plant can be described as follows

$$
\left\{\begin{array}{l}
{\left[\begin{array}{c}
\dot{x}_{s} \\
\overline{\bar{e}}
\end{array}\right]=\left[\begin{array}{cc}
A_{s} & M_{s} B \tilde{G} C \\
0 & \bar{S}^{-1}\left(\bar{A}-\left(1+\alpha_{0}\right) \bar{L}_{p} \bar{C}\right)
\end{array}\right]\left[\begin{array}{c}
x_{s} \\
\bar{e}
\end{array}\right]} \\
+\left[\begin{array}{cc}
M_{s} & 0 \\
0 & \bar{S}^{-1}
\end{array}\right]\left[\begin{array}{cc}
M_{s} & 0 \\
0 & \bar{S}^{-1}
\end{array}\right] \\
+\left[\begin{array}{c}
\phi(t, x, u) \\
\tilde{\phi}
\end{array}\right]+\left[\begin{array}{c}
B_{d} \\
-\tilde{B}_{d}
\end{array}\right] d+\left[\begin{array}{c}
0 \\
-\tilde{W}
\end{array}\right] f^{(q)} \\
x_{f}=M_{f}\left[\phi(t, x, u)+B_{d} d+B \bar{G} C \bar{e}\right]
\end{array}\right.
$$

From the well-known contraction mapping theory, the closed-loop system (22) is causal and there exists a unique solution for $x_{f}$ in the static equation in terms of $x_{s}, u, d$ and $\bar{e}$. To show the existence and uniqueness of $x_{s}$ in the dynamic equation of (22) we only need to show $J\left(t, u, x_{s}, x_{f}\right)$ is Lipschitz with respect to $x_{s}$.

\section{Consider}

$$
\left\|J\left(t, u, x_{s 1}, x_{f 1}\right)-J\left(t, u, x_{s 2}, x_{f 2}\right)\right\| \leq
$$

$\sqrt{2}\left\|\phi\left(t, N_{s} x_{s 1}+N_{f} x_{f 1}, u\right)-\phi\left(t, N_{s} x_{s 2}+N_{f} x_{f 2}, u\right)\right\|$ and

$$
\begin{array}{r}
\left\|J\left(t, u, x_{s 1}, x_{f 1}\right)-J\left(t, u, x_{s 2}, x_{f 2}\right)\right\| \leq \\
\sqrt{2} \frac{\theta \sqrt{1+\epsilon}}{\sqrt{1+\epsilon}-1}\left\|N_{s}\right\| x_{s 1}-x_{s 2} \|
\end{array}
$$

which implies $J\left(t, u, x_{s}, x_{f}\right)$ is Lipschitz with respect to $x_{s}$. Thus, the existence and uniqueness of $x_{s}$ in the dynamic equation of (22) has been verified. As a result, the equivalent plant (9) is causal and solvable. ting

(ii) The proof of the asymptotic stability: Let-

$$
V_{c}(x)=x^{\prime} E^{\prime} P x=x^{\prime} P^{\prime} E x
$$

and taking the derivative and using (9) and (11), one has

$$
\dot{V}_{c}(x) \leq x^{\prime} \Lambda x+2 x^{\prime} P^{\prime} B \bar{G} C \bar{e}+x^{\prime} P^{\prime} B_{d} d .
$$

Let

$$
V_{h}(x, \bar{e})=V_{c}(x)+\epsilon_{h} V_{0}(\bar{e})
$$

with

$$
\dot{V}_{0}(\bar{e}) \leq-\nu\|\bar{e}\|^{2}
$$

and

$$
V_{h}(x, \bar{e}) \leq e^{-\beta_{h} t / \alpha_{h}} V_{h}(x(0), \bar{e}(0))
$$

Noticing that

$$
V_{h}(x, \bar{e})=\left[\begin{array}{ll}
x_{s}^{\prime} & \bar{e}^{\prime}
\end{array}\right]\left[\begin{array}{cc}
P_{11} & 0 \\
0 & \epsilon_{h} \bar{P}
\end{array}\right]\left[\begin{array}{c}
x_{s} \\
\bar{e}
\end{array}\right]
$$

and choosing

$$
\psi=\lambda_{\min }\left[\begin{array}{cc}
P_{11} & 0 \\
0 & \epsilon_{h} \bar{P}
\end{array}\right]
$$

equations (29), (30) and (31) imply that

$$
|| \begin{gathered}
x_{s} \\
\bar{e}
\end{gathered} \mid \leq \sqrt{\frac{V_{h}(x(0), \bar{e}(0))}{\psi}} e^{-\beta_{h} t / 2 \alpha_{h}}
$$

Hence $x_{s} \rightarrow 0$ and $\bar{e} \rightarrow 0$ as $t \rightarrow \infty$. According to the output equation of (20) or (22) and under zero disturbances, one has

$$
\left\|x_{f}\right\| \leq \frac{\sqrt{1+\epsilon}}{\sqrt{1+\epsilon}-1}\left(\left\|\theta \left|\left\|| | N _ { s } \left|\left\||| x_{s}\right\|+\|B \bar{G} C|\||| \bar{e}\|) .\right.\right.\right.\right.\right.
$$

Since $x_{s} \rightarrow 0$ and $\bar{e} \rightarrow 0$ as $t \rightarrow \infty$, the equation (33) implies $x_{f} \rightarrow 0$ when $t \rightarrow \infty$. Therefore, the plant (20) or (22) is asymptotically stable, and equivalently the plant (9) is asymptotically stable.

(iii) The proof of the guaranteed performance index: Define

$$
H=\dot{V}_{h}(x, \bar{e})+y^{\prime} y-\lambda^{2} d^{\prime} d
$$

Using (9)and (26), one can derive that

$$
H \leq x_{d}^{\prime} \bar{\Omega} x_{d}+\epsilon\|x\|\|\bar{e}\|+\epsilon_{h} \dot{V}_{0}(\bar{e}),
$$

where $x_{d}=\left[x^{\prime} d^{\prime}\right]^{\prime}, \bar{\Omega}=\left[\begin{array}{cc}\Omega & P^{\prime} B_{d} \\ B_{d}^{\prime} & -\lambda^{2} I\end{array}\right]$

$$
\Omega=\Lambda+C^{\prime} C
$$

$\Lambda$ and $\epsilon_{0}$ are defined as before. Applying the Schur complement to (13), we have equivalently $\bar{\Omega}<0$. We denote

$$
\nu_{d}=\lambda_{\min }(-\bar{\Omega})
$$

Substituting (28) and (37) into (35), one has

$$
H \leq-\nu_{d}\left\|x_{d}\right\|^{2}+\epsilon_{0}\left\|x \left|\||| \bar{e}\|-\nu_{0} \epsilon_{h}\|\bar{e}\|^{2}\right.\right.
$$

and choosing $\epsilon_{h}>\epsilon_{0}^{2} / \nu_{d c} \nu_{o}$ with $\nu_{d c}=$ $\min \left(\nu_{c}, \nu_{d}\right)$, we have

$$
H \leq-\frac{\nu_{d}}{2}\left\|x_{d}\right\|^{2}-\frac{\nu_{0} \epsilon_{h}}{2}\|\bar{e}\|^{2}
$$

Under zero initial conditions and from (34) and (39), we have

$$
\int_{0}^{T f}\left(y^{\prime} y-\lambda^{2} d^{\prime} d\right) \tau \leq \int_{0}^{T f} H d \tau \leq 0
$$

which means that (10) holds. This completes the proof.

Remark 1 It is noticed that (13) is nonlinear matrix inequality, we thus have a continuous interest to transform (13) into the LMI form.

Theorem 2 The closed-loop system (9) is solvable, causal and asymptotically stable, and $\|y\|_{T_{f}} \leq \lambda\|d\|_{T_{f}}$ if there exists an non singular $Q \in \Re^{n \times n}$ and a matrix $Y \in \Re^{m \times n}$ such that

$$
\left[\begin{array}{cccc}
Q^{\prime} E^{\prime}=E Q \geq 0 \quad(41) \\
(A Q+B Y)^{\prime}+A Q+B Y & Q^{\prime} & Q^{\prime} C^{\prime} & B_{d} \\
Q & -I & 0 & 0 \\
C Q & 0 & -I & 0 \\
B_{d}^{\prime} & 0 & 0 & \lambda^{2} I
\end{array}\right]<0 \quad(42)
$$

Furthermore, if a feasible solution $(Q, Y)$ exists in the above LMIs, the output feedback gain can be $G C=Y Q^{-1}$.

Proof:

Pre-multipliying $P^{\prime-1}$ and post-multiplying $P^{-1}$ on the inequalities (12) and (13), and letting 
$P^{-1}=Q, G C P^{-1}=G C Q=Y$, then using the Schur complement, the inequalities (41) and (42) can be obtained immediately. This completes the proof.

Clearly, the equation (41) is a $L M I$, but not a strictly $L M I$. Thus, we will make a further development to give modified conditions in strictly $L M I$ forms convenience of calculation.

Lemma 3 (Ibrir, 2004) For any given vectors $\alpha$, $\beta$ and a positive definite matrix $P$ with compatible dimension, one has

$$
\alpha^{\prime} \beta+\beta^{\prime} \alpha \leq \alpha^{\prime} P \alpha+2 \beta^{\prime} P^{-1} \beta
$$

Lemma 4 (Gao and Ding, 2007) All $Z \in R^{n \times}$ satisfying

$$
Z^{\prime} E^{\prime}=E Z \geq 0
$$

can be parameterized as

$$
Z=W E^{\prime}+E_{q}^{\dagger} Q
$$

where $W \geq 0 \in \Re^{n \times n} Q \in \Re^{(n-q) \times n}$ are parameter matrices; $E_{q}^{\top} \in \Re^{n \times(n-q)}$ is a matrix such that $E E_{q}^{\top}=0$ and $\operatorname{rank}\left(E_{q}^{\top}\right)=n-\operatorname{rank}(E)=n$ - $q . \quad$ Fur thermore, when $Z$ is nonsingular, $W>0$.

Theorem 5 The closed-loop system (9) is solvable, causal and asymptotically stable, and $\|y\|_{T_{f}} \leq \lambda\|d\|_{T_{f}}$ if there exists an positive definite matrix $W \in \Re^{n \times n}$, and matrices $S \in \Re^{(n-q) \times n}$ $Y \in \Re^{m \times n}$ such that

$$
\left[\begin{array}{cccc}
\Lambda_{a_{11}} & \left(\Lambda_{a_{12}}\right)^{\prime} & \left(\Lambda_{a_{13}}\right)^{\prime} & B_{d} \\
\Lambda_{a_{12}} & -I & 0 & 0 \\
\Lambda_{a_{13}} & 0 & -I & 0 \\
B_{d}^{\prime} & 0 & 0 & -\lambda^{2} I
\end{array}\right]<0
$$

where $\Lambda_{a_{11}}=\left(A W E^{\prime}+A E_{q}^{\top} S+B Y\right)^{\prime}+A W E^{\prime}+$ $A E_{q}^{\top} S+B Y, \Lambda_{a_{12}}=W E^{\prime}+E_{q}^{\top} S, \Lambda_{a_{13}}=$ $C W E^{\prime}+C E_{q}^{\top} S, E_{q}^{\top} \in \Re^{n \times n-q}$ is a matrix such that $E E_{q}^{\top}=0$ and $\operatorname{rank}\left(E_{q}^{\top}\right)=n-\operatorname{rank}(E)=$ $n-q$. Furthermore, if a feasible solution $(W, S, Y)$ exists in the LMI (46), the output feedback gain can be computed as $G C=Y\left(W E^{\prime}+E_{q}^{\top} S\right)^{-1}$.

\section{Proof:}

Based in Theorem (2) and Lemma (4) the result holds immediately.

\section{CONCLUDING REMARKS}

For Lipschitz nonlinear descriptor systems with bounded input disturbances, by solving a Lyapunov equation, a robust state-space observer was proposed in (Gao and Ding, 2007). In this paper were presented the approach is developed for nonlinear Lipschitz descriptor systems, where the solvability, causality, asymptotic stability and performance are guaranteed. The main result provided condition for the design the output feedback controller, was presented using an LMI approach, where the theorem for existence the output feedback matrix was presented.

\section{References}

Alexandridis, A. T. and Paraskevopoulos, P. N. (1996). A new approach to eigenstruture assignment by output feedback, IEEE Trans. Automatic. Control 41(1): 1046-1050. DOI: $10.1109 / 9.508914$

Castelan, E. B., Hennet, J.-C. and Llanos Villarreal, E. R. (2003). Quadratic characterization and use of output stabilizable subspaces, IEEE Trans. Automatic. Control 48(4): 654600. DOI: 10.1109/TAC.2003.809774

Chen, Y., Weng, Z. and Shi, S. (2003). Robust fault diagnosis for nonlinear differencealgebraic system, International Journal of Control 76(1): 1560-1569. DOI: 10.1080/00207170310001605034

Dai, L. (1989). Singular Control System, SpringerVerlag. DOI: 10.1007/BFb0002475

Fletcher, L. R. (1988). Eigenstructure assingment by output feedback in descriptor systems, IEE Proceedings 135(4): 302-308.

Gao, Z. and Ding, S. X. (2007). Actuator fault robust estimation and fault-tolerant control for a class of nonlinear descriptor system, Automatica 43(1): 912-920.

DOI: 10.1016/j.automatica.2006.11.018

Gao, Z. and Ho, D. W. C. (2006). State / noise estimator for descriptor systems with application to sensor fault diagnosis, IEEE Transactions on Signal Processing 54(1): 1316-1326.

Ibrir, S. (2004). Robust state estimation with qintegral observers, Proceedings of the American Control conference, Salvador/Brasil, pp. 3466-3471.

Vemuri, A. T., Polycarpou, M. M. and Circ, A. R. (2001). Fault diagnosis of differential-algebraic systems, IEEE Transactions on Systems Man Cybernatics :Part A 31(1): 143-151. DOI: 10.1109/3468.911372

Verghese, G. C., Lèvy, B. C. and Kailath, T. (1981). A general state space for singular systems, IEEE Trans. Automatic. Control 26: 811-831. 